\title{
Highly Diastereoselective Ring-Opening Reactions of Chiral Acetals with Secondary or Sterically Hindered Grignard Reagents
}

\author{
Tien-Min Yuan, Sue-Min Yeh, Yu-Tsai Hsieh, and Tien-Yau Luh* \\ Department of Chemistry, National Taiwan University, Taipei, Taiwan 106, Republic of China
}

Received August 15, $1994^{\otimes}$

1,4-Di-tert-alkoxy-(2S,3S)-2,3-butanediols 2 were obtained from the reactions of $2 S, 3 S$-threitol bisketals with Grignard reagents. The reactions of benzylic acetals 3 , prepared from 1,4-di-tertalkoxy-(2S,3S)-2,3-butanediols and aromatic aldehydes, with aryl or secondary or sterically hindered Grignard reagents give the corresponding ring-opening products 4 in high diastereoselectivity.

There has been an increasing interest in the stereoselective ring opening of acetals by means of nucleophiles. ${ }^{1-4}$ Most of these approaches involve the preferential complex formation of acetals with a Lewis acid and subsequent invertive substitution by nucleophiles (Scheme $1)^{2}$ The retentive ring cleavage of acetals has been investigated only briefly, mostly related to the reductive ring opening with metal hydrides. ${ }^{3,4}$ Recently, Yamamoto and his co-workers reported the highly diastereoselective retentive alkylation of chiral acetals using novel organoaluminum reagents. ${ }^{4}$ However, only methyl- or primary alkylaluminum reagents can be used. We now wish to describe our investigations on the highly diastereoselective reactions of secondary or sterically bulky Grignard reagents with chiral acetals prepared from aldehydes and tunable chiral diols.

\section{Results and Discussion}

We recently reported a chelation-assisted regioselective alkylative cleavage of cyclic ketals with Grignard reagents. ${ }^{5}$ By using this strategy, 1,4-di-tert-alkoxy-(2S,3S)2,3-butanediols 2 were easily accessible from the corresponding L-tartaric acid-based bisketals 1. ${ }^{6}$ Diols 2 might demonstrate certain unique properties to serve as a ligand in asymmetric reactions. First, the size of the alkoxy substituents can be tuned. Second, the oxygen atom in the alkoxy substituent can act as an additional ligand for complexation with the metallic species which would result in the enhancement of the stereoselectivity of the reaction. Such complexation has been shown to be extremely important to direct the regioselective alkylative ring opening of acetonides. ${ }^{5}$ On the basis of this

- Abstract published in Advance ACS Abstracts, November 15, 1994. (1) For a recent review, see: Alexakis, A.; Mangeney, P. Tetrahedron: Asymmetry 1990, 1, 477.

(2) (a) Corcoran, R. C. Tetrahedron Lett. 1990, 31, 2101. (b) Bartlett, P. A. Johnson, W. S.; Elliott, J. D. J. Am. Chem. Soc. 1983, 105, 2088. (c) McNamara, J. M.; Kishi, Y. J. Am. Chem. Soc. 1982, 104, 7371. (d) Johnson, W. S.; Crackett, P. H.; Elliott, J. D.; Jagodzinski, J. J.; Lindell,

S. D.; Natarajan, S. Tetrahedron Lett. 1984, 25, 3947.

(3) (a) Richter, W. J. J. Org. Chem. 1981, 46, 5119. (b) Ishihara, K.; Mori, A.; Arai, I.; Yamamoto, H. Tetrahedron Lett. 1986, 26, 983. (c) Mori, A.; Fujiwara, J.; Maruoka, K.; Yamamoto, H. J. Organomet. Chem. 1985, 285, 83. (d) Mori, A.; Fujiwara, J.; Maruoka, K.; Yamamoto, H. Tetrahedron Lett. 1983, 24, 4581.

(4) (a) Ishihara, K.; Hanaki, N.; Yamamoto, H. J. Am. Chem. Soc. 1991, 113, 7074. (b) Ishihara, K.; Hanaki, N.; Yamamoto, H. J. Am. Chem. Soc. 1993, 115, 10695.

(5) Cheng, W.-L.; Yeh, S.-M.; Luh, T.-Y. J. Org. Chem. 1993, 58, 5576. Chen, Y.-H.; Luh, T.-Y.; Lee, G.-H.; Peng, S.-M. J. Chem. Soc., Chem. Commun. 1994, 2369.

(6) (a) Feit, P. W. Chem. Ber. 1963, 96, 712. (b) Mash, E. A.; Nelson, K. A. Van Deusen, S.; Hemperly, S. B. Org. Synth. 1989, 68, 92. (c) Mash, E. A.; Nelson, K. A. Tetrahedron 1987, 43, 679. (d) Mash, E. A.; Hemperly, S. B.; Nelson, K. A.; Heidt, P. C.; Van Deusen, S. J. Org. Chem. 1990, 55, 2045. (e) Tamura, Y.; Kondo, H.; Annoura, H. Tetrahedron Lett. 1986, 27, 81. (f) Tamura, Y.; Ko, T.; Kondo, H.; Annoura, H. Tetrahedron Lett. 1986, 27, 2117.

0022-3263/94/1959-8192\$04.50/0

\section{Scheme 1}

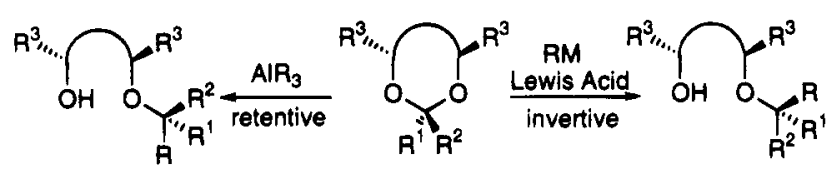

$R^{1}<R^{2}, R=$ Me or primary alkyl

conjecture, we felt that this advantage could be made use of in the diastereoselective ring-opening reactions of chiral acetals 3 with Grignard reagents.

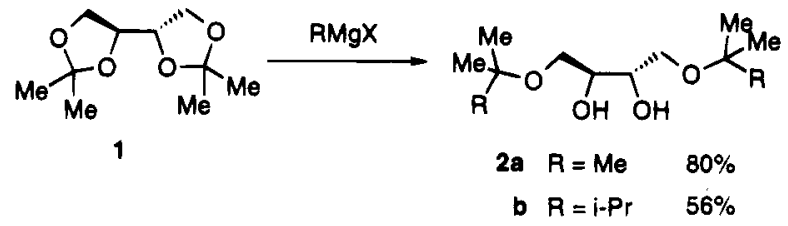

Acetals 3 were conveniently prepared according to a modified procedure. ${ }^{7}$ Treatment of $(4 S, 5 S)-3$ with the Grignard reagent in refluxing benzene solution afforded the corresponding ring opening products 4 in good yields (Table 1).

The diastereoselectivity was determined by HPLC and/ or by ${ }^{1} \mathrm{H}$ NMR, and the major isomer of each product was isolated by preparative HPLC. The absolute configuration of the the major isomer $\left(\left(2 S, 3 S, 1^{\prime} S\right)-4\right)$ of the ringopening products 4 was determined by degradation ${ }^{2 b, c}$ leading to the corresponding chiral alcohols $(S)-5$.

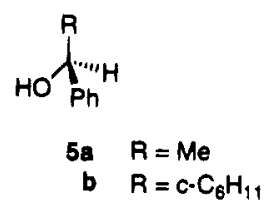

Interestingly, the diastereoselectivities for the reactions of 3a with $\mathrm{MeMgI}$ in the presence and in the absence of $\mathrm{TiCl}_{2}(\mathrm{OPr}-\mathrm{i})_{2}$ were opposite (eq 1). Presum-

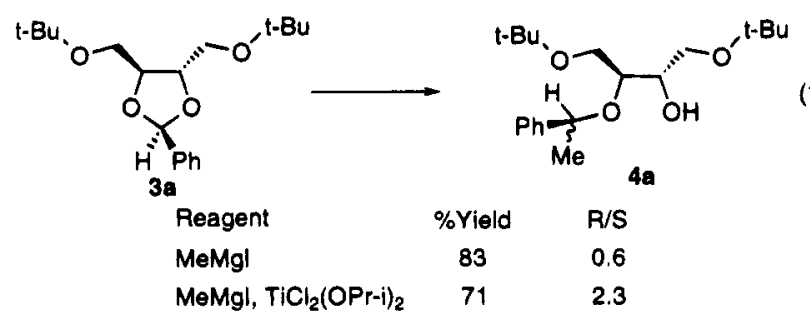

(7) Chan, T. H.; Brook, M. A.; Chaly, T. Synthesis 1983, 203. 
Table 1. Reaction of Acetals 3 with Grignard Reagents

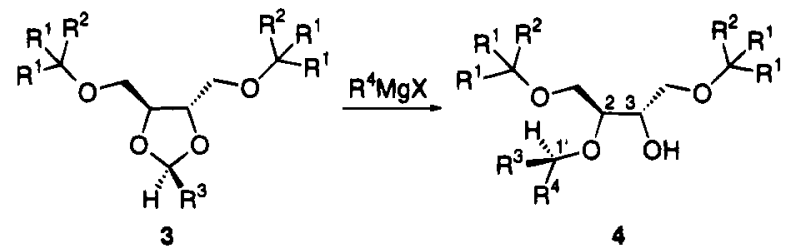

\begin{tabular}{|c|c|c|c|c|c|c|c|}
\hline & & & & & & 4 & \\
\hline try & 3 & $R^{1}$ & $R^{2}$ & $R^{3}$ & $R^{4}$ & 4 (\% Yield) & de $\%$ \\
\hline 1 & a & Me & $\mathrm{Me}$ & $\mathrm{Ph}$ & Me & (83) & 26 \\
\hline 2 & a & Me & $\mathrm{Me}$ & $\mathrm{Ph}$ & i-Pr & b (77) & 48 \\
\hline 3 & b & Me & i-Pr & $\mathrm{m}-\mathrm{MeOC}_{6} \mathrm{H}_{4}$ & i-Pr & $c(79)$ & 71 \\
\hline 4 & c & $H$ & H & $\mathrm{Ph}$ & $c-\mathrm{C}_{5} \mathrm{H}_{9}$ & $d(82)$ & 64 \\
\hline 5 & a & Me & $\mathrm{Me}$ & $\mathrm{Ph}$ & $\mathrm{c}-\mathrm{C}_{5} \mathrm{H}_{9}$ & e (83) & 96 \\
\hline 6 & d & $\mathrm{Me}$ & Me & p- $-\mathrm{PhC}_{6} \mathrm{H}_{4}$ & $\mathrm{C}-\mathrm{C}_{5} \mathrm{H}_{9}$ & $f(84)$ & 95 \\
\hline 7 & - & Me & i-Pr & $\mathrm{p}-\mathrm{PhC}_{6} \mathrm{H}_{4}$ & $c-\mathrm{C}_{5} \mathrm{H}_{9}$ & $g(78)$ & 96 \\
\hline 8 & 1 & $\mathrm{Me}$ & $\mathrm{Me}$ & $\mathrm{p}-\mathrm{BrC}_{6} \mathrm{H}_{4}$ & c- $\mathrm{C}_{5} \mathrm{H}_{9}$ & $h(77)$ & 95 \\
\hline 9 & 9 & $\mathrm{Me}$ & $\mathrm{Me}$ & $\mathrm{m}-\mathrm{MeOC}_{6} \mathrm{H}_{4}$ & $c-\mathrm{C}_{6} \mathrm{H}_{11}$ & $I(80)$ & $>98^{\mathrm{a}}$ \\
\hline 10 & a & $\mathrm{Me}$ & $\mathrm{Me}$ & $\mathrm{Ph}$ & c- $\mathrm{C}_{6} \mathrm{H}_{11}$ & I (84) & $>98^{\mathrm{A}}$ \\
\hline 11 & 9 & Me & Me & $\mathrm{m}-\mathrm{MeOC}_{6} \mathrm{H}_{4}$ & $\mathrm{Ph}$ & $k(73)$ & $>98^{\mathrm{a}}$ \\
\hline 12 & 9 & $\mathrm{Me}$ & Me & $\mathrm{m}-\mathrm{MeOC}_{6} \mathrm{H}_{4}$ & $\mathrm{Me}_{3} \mathrm{CCH}_{2}$ & I (81) & 94 \\
\hline
\end{tabular}

a The minor diastereomer was not detected.

ably, the titanium reagent competes with the Grignard reagent for complexation resulting in the discrepancy in selectivity. It is noteworthy that the selectivity of such titanium-promoted reaction paralleled to those in the other substrates using similar conditions. ${ }^{2 d}$

Several interesting features about the ring-opening reactions with the Grignard reagent are worthy of comment. First, the size of the substituent affects the diastereoselectivity of the reaction. Thus, the percent diastereomeric excess increased from 64 to $96 \%$ when the alkoxy subtituent changed from methyl to thexyl when cyclopentyl Grignard reagent was employed (entries 4-7). Second, the highest diastereoselectivities of this ring opening process were observed when sterically hindered or secondary cyclic Grignard reagents were employed (entries 4-12). To the best of our knowledge, all literature procedures use methyl or primary alkyl organometallic reagents, and no reports are known to employ sterically hindered or secondary organometallic reagents to transfer the chirality in the nucleophilic reactions of an acetal group.

As mentioned earlier, the oxygen atom in the alkoxy substituent can act as an additional ligand for complexation with the metallic species. Accordingly, complexation between the substrate 3 and the Grignard reagent may occur; and with bulky Grignard reagent, intermediate $6 \mathbf{a}$ would be more stable than its stereoisomer $\mathbf{6 b}$.

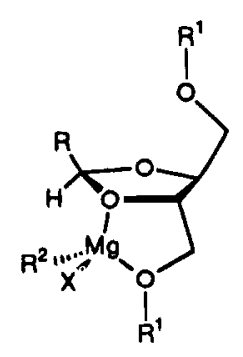

6a

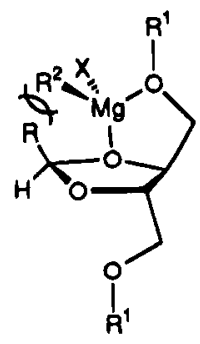

6b
Although the actual mode of the ring opening reaction of acetals is not yet clear, retentive displacement of the carbon-oxygen bond by an alkyl group from intermediate $6 \mathbf{a}$ is speculated. ${ }^{8}$

In order to clarify the validity of this conjecture, the reaction of $(4 S, 5 S)-7$ which does not have the oxygen atom for chelation, with $\mathrm{MeMgI}$ under similar conditions gave 8 in $82 \%$ yield with $30 \%$ de in favor of $\left(2 S, 3 S, 1^{\prime} R\right)-8$ (eq 2). The selectivity was just opposite to our observa-

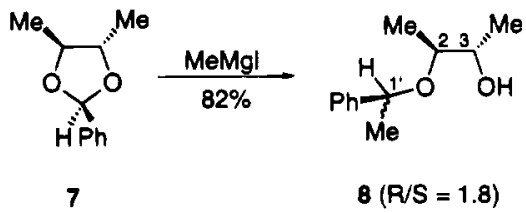

tion as described in Table 1. The discrepancy between the reaction in eq 2 and those in Table 1 suggested that the chelation intermediate $\mathbf{6 a}$ may be involved in the reaction of 3 with Grignard reagents leading to the displacement of a carbon-oxygen bond by a carboncarbon bond.

In summary, we have demonstrated the unprecedented example of the highly diastereoselective ring opening reaction of cyclic acetals with secondary or sterically bulky Grignard reagents using tunable diol auxiliaries. Our procedure compliments with the existing methods ${ }^{1-3}$ where only primary alkyl nucleophiles can proceed such carbon-carbon bond formation reactions. Other asymmetric synthetic applications of tunable diols 2 are currently under investigation in our laboratory.

\section{Experimental Section}

General Procedure for the Preparation of Diol 2. To a benzene $(250 \mathrm{~mL})$ solution of $1(25 \mathrm{mmol})$ was added excess Grignard reagent. The mixture was refluxed for $20 \mathrm{~h}$. Saturated $\mathrm{NH}_{4} \mathrm{Cl}$ solution $(125 \mathrm{~mL})$ was added, and the mixture was extracted with ether $(100 \mathrm{~mL} \times 3)$. The organic layer was washed with brine $(250 \mathrm{~mL})$ and dried $\left(\mathrm{MgSO}_{4}\right)$. The solvent was removed in vacuo, and the residue was distilled or chromatographed on silica gel (EtOAc/Hex $=1 / 4)$ to give 2 .

Diol 2a. Following the general procedure, the reaction of $1 \mathrm{a}^{6 \mathrm{a}}(10.1 \mathrm{~g}, 50.0 \mathrm{mmol})$ and $\mathrm{MeMgI}(80 \mathrm{~mL}$ of a $2 \mathrm{M}$ solution in ether, $160 \mathrm{mmol})$ in benzene $(500 \mathrm{~mL})$ gave $2 \mathrm{a}(9.36 \mathrm{~g}$, $80 \%)$ : bp $98-100^{\circ} \mathrm{C}(1 \mathrm{mmHg}) ;[\alpha]^{30} \mathrm{D}=+4.8^{\circ}\left(\mathrm{c} 7.2, \mathrm{CHCl}_{3}\right)$; IR (neat) $v 3462,2973,2932,2876,1475,1390,1365,1235$, $1196,1087,1020,883 \mathrm{~cm}^{-1} ;{ }^{1} \mathrm{H} \mathrm{NMR}\left(\mathrm{CDCl}_{3}, 300 \mathrm{MHz}\right) \delta 1.18$ (s, $18 \mathrm{H}$ ), 3.03 (d, $J=4.8 \mathrm{~Hz}, 2 \mathrm{H}$ ), 3.48 (dd, $J=9.2,5.3 \mathrm{~Hz}$, $2 \mathrm{H}$ ), 3.52 (dd, $J=9.2,4.7 \mathrm{~Hz}, 2 \mathrm{H}), 3.73-3.81(\mathrm{~m}, 2 \mathrm{H}) ;{ }^{13} \mathrm{C}$ $\mathrm{NMR}\left(\mathrm{CDCl}_{3}, 75 \mathrm{MHz}\right) \delta 27.4,64.1,70.9,73.4 ; \mathrm{MS}(20 \mathrm{eV})$ $\mathrm{m} / z$ (rel intensity) $235(\mathrm{M}+1,100), 179(55), 129(6), 123(18)$,

(8) An ion pair mechanism has been proposed for the invertive Lewis acid catalyzed reactions of acetals with allylsilanes (cf. Denmark, $S$. E.; Almstead, N. G. J. Am. Chem. Soc. 1991, 113, 8089). In line with this argument, complexation of magnesium ion with the pro- $R$ oxygen would give intermediate $6 \mathrm{c}$ which might undergo invertive ring opening from the $S i$-face to give the desired selectivity as shown in Table 1. However, the opposite selectivity from the reaction of 7 (eq 2) could not fit into this model.

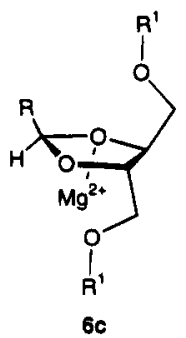


$105(3), 73$ (3), 57 (25); HRMS calcd for $\mathrm{C}_{12} \mathrm{H}_{27} \mathrm{O}_{4}(\mathrm{M}+1)$ 235.1909 , found 235.1908 .

Diol 2b. Following the general procedure, the reaction of $1 \mathrm{a}(2.02 \mathrm{~g}, 10.0 \mathrm{mmol})$ and $\mathrm{Me}_{2} \mathrm{CHMgBr}(50 \mathrm{~mL}$ of a $1.2 \mathrm{M}$ solution in ether, $60 \mathrm{mmol})$ in benzene $(120 \mathrm{~mL})$ gave $\mathbf{2 b}(1.63$ g, 56\%): bp $124-126{ }^{\circ} \mathrm{C}(1 \mathrm{mmHg}) ;[\alpha]^{30} \mathrm{D}=+5.5^{\circ}(c 4.2$, $\mathrm{CHCl}_{3}$ ); IR (neat) v 3443, 2973, 2937, 2878, 1471, 1391, 1088, $903 \mathrm{~cm}^{-1}$; ${ }^{1} \mathrm{H}$ NMR $\left(\mathrm{CDCl}_{3}, 300 \mathrm{MHz}\right) \delta 0.84(\mathrm{~d}, J=6.7 \mathrm{~Hz}$, $12 \mathrm{H}$ ), 1.07 (s, $12 \mathrm{H}$ ), 1.77 (septet, $J=6.7 \mathrm{~Hz}, 2 \mathrm{H}$ ), 3.00 (d, $J$ $=4.8 \mathrm{~Hz}, 2 \mathrm{H}), 3.44(\mathrm{dd}, J=9.0,5.1 \mathrm{~Hz}, 2 \mathrm{H}), 3.49(\mathrm{dd}, J=$ $9.0,4.8 \mathrm{~Hz}, 2 \mathrm{H}), 3.72-3.79(\mathrm{~m}, 2 \mathrm{H}) ;{ }^{13} \mathrm{C} \mathrm{NMR}\left(\mathrm{CDCl}_{3}, 75\right.$ MHz) $\delta 17.4,22.0,35.7,63.1,71.0,77.8 ; \mathrm{MS}(20 \mathrm{eV}) \mathrm{m} / z$ (rel intensity) $291(\mathrm{M}+1,88), 249(17), 207(100), 206(28), 165$ (20), $163(18), 147(9), 123(34), 105(16), 85(15)$; HRMS calcd for $\mathrm{C}_{16} \mathrm{H}_{35} \mathrm{O}_{4}(\mathrm{M}+1) 291.2535$, found 291.2517.

General Procedure for the Preparation of Acetal 3. To a $\mathrm{CH}_{2} \mathrm{Cl}_{2}(10 \mathrm{~mL})$ solution of diol $2(1.0 \mathrm{mmol})$ and benzaldehyde $(1.0 \mathrm{mmol})$ was added TMSCl $(4.0 \mathrm{mmol})$. The mixture was stirred for $24 \mathrm{~h}$. Sodium hydroxide solution (10\%, $10 \mathrm{~mL}$ ) was added, and the mixture was extracted with ether $(15 \mathrm{~mL} \times 3)$. The organic layer was washed with brine $(25$ $\mathrm{mL}$ ) and dried $\left(\mathrm{MgSO}_{4}\right)$. The solvent was evaporated in vacuo, and the residue was chromatographed on silica gel (5\% ethyl acetate in hexane) to give the corresponding acetal $\mathbf{3}$.

Acetal 3a. Following the general procedure, the reaction of $2 \mathrm{a}(1.17 \mathrm{~g}, 5.0 \mathrm{mmol})$, benzaldehyde $(530 \mathrm{mg}, 5.0 \mathrm{mmol})$, and TMSCl $(2.5 \mathrm{~mL}, 20 \mathrm{mmol})$ in $\mathrm{CH}_{2} \mathrm{Cl}_{2}$ gave $3 \mathrm{a}(757 \mathrm{mg}$, $47 \%$ ): $[\alpha]^{25}{ }_{\mathrm{D}}=+4.7^{\circ}$ (c 30, $\mathrm{CHCl}_{3}$ ); IR (neat) $v 2974,2931$, $2873,1461,1388,1365,1196,1090,1068,756,699 \mathrm{~cm}^{-1} ;{ }^{1} \mathrm{H}$ NMR $\left(300 \mathrm{MHz}, \mathrm{CDCl}_{3}\right) \delta 1.19(\mathrm{~s}, 9 \mathrm{H}), 1.20(\mathrm{~s}, 9 \mathrm{H}), 3.51-$ $3.65(\mathrm{~m}, 4 \mathrm{H}), 4.03(\mathrm{dt}, J=6.6,5.0 \mathrm{~Hz}, 1 \mathrm{H}), 4.08-4.14(\mathrm{~m}, 1$ $\mathrm{H}), 5.93(\mathrm{~s}, 1 \mathrm{H}), 7.31-7.37(\mathrm{~m}, 3 \mathrm{H}), 7.46-7.50(\mathrm{~m}, 2 \mathrm{H}) ;{ }^{13} \mathrm{C}$ NMR $\left(75 \mathrm{MHz} \mathrm{CDCl}_{3}\right) \delta 27.4,62.7,63.1,73.2,78.7,79.3$, $104.0,126.8,128.1,129.1,137.9 ; \mathrm{MS}(20 \mathrm{eV}) \mathrm{m} / z$ (rel intensity) $323(\mathrm{M}+1,12), 267(15), 265(66), 235(6), 211(28), 209(10)$, 179 (53), 107 (12), 105 (10), 87 (7), 69 (11), 57 (100); HRMS calcd for $\mathrm{C}_{19} \mathrm{H}_{30} \mathrm{O}_{4} 322.2144$, found 322.2141 .

Acetal 3b. Following the general procedure, the reaction of $2 \mathrm{~b}(290 \mathrm{mg}, 1.0 \mathrm{mmol}$ ), 3-methoxybenzaldehyde (136 $\mathrm{mg}$, $1.0 \mathrm{mmol})$ and TMSCl $(0.5 \mathrm{~mL}, 4.0 \mathrm{mmol})$ in $\mathrm{CH}_{2} \mathrm{Cl}_{2}$ gave $3 \mathrm{~b}$ $(212 \mathrm{mg}, 52 \%):[\alpha]^{27} \mathrm{D}=+5.8^{\circ}$ (c 4.0, $\mathrm{CHCl}_{3}$ ); IR (neat) $\nu 2971$, $2878,1606,1591,1467,1366,1265,1197,1171,1094,903,787$ $\mathrm{cm}^{-1}$; ${ }^{1} \mathrm{H}$ NMR $\left(300 \mathrm{MHz}, \mathrm{CDCl}_{3}\right) \delta 0.87(\mathrm{~d}, J=6.9 \mathrm{~Hz}, 6 \mathrm{H})$, $0.89(\mathrm{~d}, J=6.9 \mathrm{~Hz}, 6 \mathrm{H}), 1.08(\mathrm{~s}, 6 \mathrm{H}), 1.09(\mathrm{~s}, 6 \mathrm{H}), 1.80$ (septet, $J=6.9 \mathrm{~Hz}, 2 \mathrm{H}), 3.47-3.62(\mathrm{~m}, 4 \mathrm{H}), 3.79(\mathrm{~s}, 3 \mathrm{H})$, $4.06(\mathrm{dt}, J=6.2,4.7 \mathrm{~Hz}, 1 \mathrm{H}), 4.14-4.19(\mathrm{~m}, 1 \mathrm{H}), 5.91(\mathrm{~s}, 1$ $\mathrm{H}), 6.87(\mathrm{~d}, J=7.8 \mathrm{~Hz}, 1 \mathrm{H}), 7.05(\mathrm{br} \mathrm{s}, 1 \mathrm{H}), 7.06(\mathrm{~d}, J=7.8$ $\mathrm{Hz}, 1 \mathrm{H}), 7.25(\mathrm{t}, J=7.8 \mathrm{~Hz}, 1 \mathrm{H}) ;{ }^{13} \mathrm{C} \mathrm{NMR}\left(75 \mathrm{MHz}, \mathrm{CDCl}_{3}\right)$ $\delta 17.4,17.5,22.0,35.7,35.9,55.2,61.9,62.2,77.4,78.8,79.0$, $103.7,111.7,115.2,119.2,129.2,139.5,159.6 ; \mathrm{MS}(20 \mathrm{eV}) \mathrm{m} / z$ (rel intensity) $409(\mathrm{M}+1,2), 408(2), 407(2), 323(100), 281$ (32), 241 (29), 239 (14), 223 (7), 209 (12), 147 (7), 135 (8), 121 (10), 85 (52); HRMS calcd for $\mathrm{C}_{24} \mathrm{H}_{40} \mathrm{O}_{5} 408.2876$, found 408.2873

Acetal 3d. Following the general procedure, the reaction of $2 \mathrm{a}$ (468 $\mathrm{mg}, 2.0 \mathrm{mmol}$ ), 4-phenylbenzaldehyde (364 mg, 2.0 $\mathrm{mmol})$ and TMSCl $(1.0 \mathrm{~mL}, 8.0 \mathrm{mmol})$ in $\mathrm{CH}_{2} \mathrm{Cl}_{2}$ gave $3 \mathrm{~d}(446$ $\mathrm{mg}, 56 \%):[\alpha]^{27} \mathrm{D}=+11.7^{\circ}\left(\mathrm{c} 18, \mathrm{CHCl}_{3}\right.$ ); IR (neat) $v 3031,2974$, $2931,2874,1487,1364,1195,1088,834,765,698 \mathrm{~cm}^{-1} ;{ }^{1} \mathrm{H}$ NMR $\left(\mathrm{CDCl}_{3}, 300 \mathrm{MHz}\right) \delta 1.24(\mathrm{~s}, 9 \mathrm{H}), 1.25(\mathrm{~s}, 9 \mathrm{H}), 3.57-$ $3.71(\mathrm{~m}, 4 \mathrm{H}), 4.10(\mathrm{dt}, J=6.5,5.0 \mathrm{~Hz}, 1 \mathrm{H}), 4.15-4.21(\mathrm{~m}, 1$ $\mathrm{H}), 6.03(\mathrm{~s}, 1 \mathrm{H}), 7.31-7.37(\mathrm{~m}, 1 \mathrm{H}), 7.41-7.46(\mathrm{~m}, 2 \mathrm{H}), 7.57-$ $7.61(\mathrm{~m}, 6 \mathrm{H}) ;{ }^{13} \mathrm{C} \mathrm{NMR}\left(\mathrm{CDCl}_{3}, 75 \mathrm{MHz}\right) \delta 27.4,62.6,63.0$, 73.1, 78.6, 79.2, 103.7, 126.9, 127.1, 127.2, 127.3, 128.6, 136.8, $140.8,142.0 ; \mathrm{MS}(20 \mathrm{eV}) \mathrm{m} / \boldsymbol{z}$ (rel intensity) $399(\mathrm{M}+1,7)$, 398 (4), 397 (6), 341 (100), 285 (9), 255 (18), 199 (8), 181 (19), 69 (6), 57 (25); HRMS calcd for $\mathrm{C}_{25} \mathrm{H}_{34} \mathrm{O}_{4}$ 398.2457, found 398.2468 .

Acetal 3e. Following the general procedure, the reaction of $2 \mathrm{~b}$ ( $290 \mathrm{mg}, 1.0 \mathrm{mmol}$ ), 4-phenylbenzaldehyde (182 mg, 1.0 $\mathrm{mmol})$, and TMSCl $(0.5 \mathrm{~mL}, 4.0 \mathrm{mmol})$ in $\mathrm{CH}_{2} \mathrm{Cl}_{2}$ gave $3 \mathrm{e}(263$ $\mathrm{mg}, 58 \%):[\alpha]^{30} \mathrm{D}=+15.0^{\circ}$ (c 10, $\mathrm{CHCl}_{3}$ ); IR (neat) v 2972, 2876 , $1466,1366,1152,1111,1089,832,765,698 \mathrm{~cm}^{-1} ;{ }^{1} \mathrm{H}$ NMR $\left(300 \mathrm{MHz}, \mathrm{CDCl}_{3}\right) \delta 0.89(\mathrm{~d}, J=6.8 \mathrm{~Hz}, 6 \mathrm{H}), 0.90(\mathrm{~d}, J=6.8$ $\mathrm{Hz}, 6 \mathrm{H}), 1.10(\mathrm{~s}, 6 \mathrm{H}), 1.11(\mathrm{~s}, 6 \mathrm{H}), 1.81$ (septet, $J=6.8 \mathrm{~Hz}$, $2 \mathrm{H}), 3.50-3.65(\mathrm{~m}, 4 \mathrm{H}), 4.10(\mathrm{dt}, J=6.3,4.7 \mathrm{~Hz}, 1 \mathrm{H}), 4.17-$ $4.23(\mathrm{~m}, 1 \mathrm{H}), 5.99(\mathrm{~s}, 1 \mathrm{H}), 7.30-7.36(\mathrm{~m}, 1 \mathrm{H}), 7.39-7.45(\mathrm{~m}$, $2 \mathrm{H}), 7.54-7.60(\mathrm{~m}, 6 \mathrm{H}) ;{ }^{13} \mathrm{C}$ NMR $\left(75 \mathrm{MHz} \mathrm{CDCl}_{3}\right) \delta 17.5$, $22.0,22.1,35.8,36.0,61.9,62.3,77.5,78.9,79.1,103.8,127.0$, $127.2,127.3,128.7,137.0,141.0,142.1 ;$ MS $(20 \mathrm{eV}) \mathrm{m} / z($ rel intensity) $455(\mathrm{M}+1,1), 454(0.7), 453(2), 411(1), 369(100)$, 327 (25), 287 (11), 285 (10), 199 (6), 181 (12), 167 (8), 85 (43), 69 (6); HRMS calcd for $\mathrm{C}_{29} \mathrm{H}_{42} \mathrm{O}_{4} 454.3083$, found 454.3085 .

Acetal 3f. Following the general procedure, the reaction of $2 \mathrm{a}(468 \mathrm{mg}, 2.0 \mathrm{mmol}$ ), 4-bromobenzaldehyde ( $370 \mathrm{mg}, 2.0$ mmol), and TMSCl $(1.0 \mathrm{~mL}, 8.0 \mathrm{mmol})$ in $\mathrm{CH}_{2} \mathrm{Cl}_{2}$ gave $3 \mathrm{f}(481$ $\mathrm{mg}, 60 \%):[\alpha]^{27} \mathrm{D}=+14.1^{\circ}\left(\mathrm{c} 10, \mathrm{CHCl}_{3}\right)$; IR (neat) $\nu 2974,2934$, $2875,1591,1484,1364,1271,1195,1091,1013,757 \mathrm{~cm}^{-1} ;{ }^{1} \mathrm{H}$

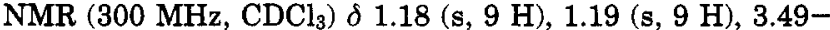
$3.61(\mathrm{~m}, 4 \mathrm{H}), 4.01(\mathrm{dt}, J=6.5,4.9 \mathrm{~Hz}, 1 \mathrm{H}), 4.07-4.13(\mathrm{~m}, 1$ $\mathrm{H}), 5.88(\mathrm{~s}, 1 \mathrm{H}), 7.36(\mathrm{~d}, J=8.4 \mathrm{~Hz}, 2 \mathrm{H}), 7.46(\mathrm{~d}, J=8.4 \mathrm{~Hz}$, $2 \mathrm{H}) ;{ }^{13} \mathrm{C} \mathrm{NMR}\left(75 \mathrm{MHz}, \mathrm{CDCl}_{3}\right) \delta 27.4,62.6,62.9,73.2,78.6$, $79.3,103.3,123.2,128.6,131.3,137.1 ; \mathrm{MS}(20 \mathrm{eV}) \mathrm{m} / z$ (rel intensity) $403\left(\mathrm{M}\left({ }^{81} \mathrm{Br}\right)^{+}+1,1.5\right), 401\left(\mathrm{M}\left({ }^{79} \mathrm{Br}\right)^{+}+1,3\right), 399$ (1.5), 345 (49), 343 (49), 291 (6), 289 (12), 287 (6), 259 (38), 257 (38), 84 (14), 69 (14), 57 (100), 49 (20); HRMS calcd for $\mathrm{C}_{19} \mathrm{H}_{28} \mathrm{O}_{4}{ }^{79} \mathrm{Br}(\mathrm{M}-1) 399.1171$, found 399.1170 ; calcd for $\mathrm{C}_{19} \mathrm{H}_{28} \mathrm{O}_{4}{ }^{81} \mathrm{Br}(\mathrm{M}-1) 401.1151$, found 401.1154 .

Acetal 3g. Following the general procedure, the reaction of $2 \mathrm{a}$ (936 mg, $4.0 \mathrm{mmol}$ ), 3-methoxybenzaldehyde $(544 \mathrm{mg}$, $4.0 \mathrm{mmol})$, and $\mathrm{TMSCl}(2.0 \mathrm{~mL}, 16 \mathrm{mmol})$ in $\mathrm{CH}_{2} \mathrm{Cl}_{2}$ gave $3 \mathrm{~g}$ $(760 \mathrm{mg}, 54 \%):[\alpha]^{26} \mathrm{D}=+6.0^{\circ}\left(\right.$ c $\left.6.7, \mathrm{CHCl}_{3}\right)$; IR (neat) $v 2974$, $2872,1604,1591,1468,1364,1284,1196,1089,878,785,695$ $\mathrm{cm}^{-1} ;{ }^{1} \mathrm{H} \mathrm{NMR}\left(300 \mathrm{MHz}, \mathrm{CDCl}_{3}\right) \delta 1.19(\mathrm{~s}, 9 \mathrm{H}), 1.20(\mathrm{~s}, 9 \mathrm{H})$, $3.50-3.64(\mathrm{~m}, 4 \mathrm{H}), 3.79(\mathrm{~s}, 3 \mathrm{H}), 4.02(\mathrm{dt}, J=6.6,5.0 \mathrm{~Hz}, 1$ $\mathrm{H}), 5.91(\mathrm{~s}, 1 \mathrm{H}), 6.86(\mathrm{~d}, J=8.0 \mathrm{~Hz}, 1 \mathrm{H}), 7.05(\mathrm{~s}, 1 \mathrm{H}), 7.06$ $(\mathrm{d}, J=7.6 \mathrm{~Hz}, 1 \mathrm{H}), 7.24(\mathrm{dd}, J=8.0,7.6 \mathrm{~Hz}, 1 \mathrm{H}) ;{ }^{13} \mathrm{C}$ NMR $\left(75 \mathrm{MHz}_{1} \mathrm{CDCl}_{3}\right.$ ) $\delta 27.4,55.1,62.6,63.0,73.1,78.6,79.2,103.7$, $111.7,115.1,119.1,129.1,139.4,159.5 ; \mathrm{MS}(70 \mathrm{eV}) \mathrm{m} / \mathrm{z}$ (rel intensity) $352(\mathrm{M}, 25), 295$ (100), 239 (20), 209 (58), 153 (21), $137(38), 135$ (79), 121 (26), 109 (31), 87 (17); HRMS calcd for $\mathrm{C}_{20} \mathrm{H}_{32} \mathrm{O}_{5} 352.2250$, found 352.2246 .

General Procedure for Reaction of Acetal 3 with Grignard Reagent. To a benzene (15 mL) solution of $3(0.25$ $\mathrm{mmol}$ ) was added Grignard reagent $(1.0 \mathrm{~mL}$ of a $1.0 \mathrm{M}$ solution in ether, $1.0 \mathrm{mmol}$ ). The mixture was refluxed for $10 \mathrm{~h}$. Saturated $\mathrm{NH}_{4} \mathrm{Cl}(15 \mathrm{~mL})$ was added, and the mixture was extracted with ether $(15 \mathrm{~mL} \times 2)$. The organic layer was washed with brine $(20 \mathrm{~mL})$ and dried $\left(\mathrm{MgSO}_{4}\right)$. The solvent was removed in vacuo, and the residue was chromatographed on silica gel $(\mathrm{EtOAc} / \mathrm{Hex}=1 / 9)$ to give a mixture of diastereomeric products. The ratio of the diastereomers was determined by HPLC on a silica gel column and/or by ${ }^{1} \mathrm{H}$ NMR. The major diastereomer was obtained by preparative HPLC on silica gel.

Reaction of 3a with MeMgI. Following the general procedure, the reaction of $3 \mathrm{a}(161 \mathrm{mg}, 0.5 \mathrm{mmol})$ and $\mathrm{MeMgI}$ $(2.0 \mathrm{~mL}$ of a $1.0 \mathrm{M}$ solution in ether, $2.0 \mathrm{mmol})$ in benzene gave $4 \mathrm{a}(140 \mathrm{mg}, 83 \%$, de $26 \%)$. (2S,3S,1'S)-4a: $[\alpha]^{27}{ }_{\mathrm{D}}=$ $-35.0^{\circ}$ (c 1.5, $\mathrm{CHCl}_{3}$ ); IR (neat) $v 3480,2974,2932,2874,1474$ $1453,1389,1364,1196,1087,1022,700 \mathrm{~cm}^{-1},{ }^{1} \mathrm{H}$ NMR $\left(\mathrm{CDCl}_{3}\right.$ $300 \mathrm{MHz}) \delta 1.03(\mathrm{~s}, 9 \mathrm{H}), 1.22(\mathrm{~s}, 9 \mathrm{H}), 1.44(\mathrm{~d}, J=6.5 \mathrm{~Hz}, 3$ $\mathrm{H}), 2.95(\mathrm{~d}, J=5.2 \mathrm{~Hz}, 1 \mathrm{H}), 3.21(\mathrm{dd}, J=9.3,4.9 \mathrm{~Hz}, 1 \mathrm{H}$ ), 3.35 (dd, $J=9.3,6.0 \mathrm{~Hz}, 1 \mathrm{H}$ ), 3.45 (dd, $J=8.9,6.4 \mathrm{~Hz}, 1 \mathrm{H}$ ), 3.51 (dd, $J=8.9,5.7 \mathrm{~Hz}, 1 \mathrm{H}), 3.55-3.59(\mathrm{~m}, 1 \mathrm{H}), 3.79-3.88$ $(\mathrm{m}, 1 \mathrm{H}), 4.61(\mathrm{q}, J=6.5 \mathrm{~Hz}, 1 \mathrm{H}), 7.21-7.36(\mathrm{~m}, 5 \mathrm{H}) ;{ }^{13} \mathrm{C}$ $\mathrm{NMR}\left(\mathrm{CDCl}_{3}, 75 \mathrm{MHz}\right) \delta 23.6,27.2,27.6,62.3,62.6,71.6,73.0$, $73.1,76.4,78.3,126.4,127.4,128.2,144.4 ; \mathrm{MS}(20 \mathrm{eV}) \mathrm{m} / \mathrm{z}$ (rel intensity) $339(\mathrm{M}+1,40), 310(5), 300(6), 233(6), 225$ (15), 144 (9), 129 (16), 126 (8), 121 (19), $117(11), 105$ (100), 57 (70); HRMS calcd for $\mathrm{C}_{20} \mathrm{H}_{34} \mathrm{O}_{4} 338.2457$, found 338.2448. $\left(2 S, 3 S, 1^{\prime} R\right)-4 \mathrm{a}:{ }^{1} \mathrm{H}$ NMR $\left(\mathrm{CDCl}_{3}, 300 \mathrm{MHz}\right) \delta 1.06(\mathrm{~s}, 9 \mathrm{H})$, $1.20(\mathrm{~s}, 9 \mathrm{H}), 1.45(\mathrm{~d}, J=6.6 \mathrm{~Hz}, 3 \mathrm{H}), 2.66(\mathrm{br} \mathrm{d}, J=3.6 \mathrm{~Hz}$, $1 \mathrm{H}$ ), 2.99 (dd, $J=9.2,4.4 \mathrm{~Hz}, 1 \mathrm{H}$ ), $3.25(\mathrm{t}, J=9.2,7.6 \mathrm{~Hz}$, $1 \mathrm{H}), 3.38(\mathrm{~m}, 1 \mathrm{H}), 3.52(\mathrm{dd}, J=9.4,5.4 \mathrm{~Hz}, 1 \mathrm{H}), 3.62(\mathrm{dd}, J$ $=9.4,5.1 \mathrm{~Hz}, 1 \mathrm{H}), 4.61(\mathrm{q}, J=6.6 \mathrm{~Hz}, 1 \mathrm{H}), 7.25-7.32(\mathrm{~m}, 5$ $\mathrm{H})$.

Reaction of 3a with $\mathrm{Me}_{2} \mathrm{CHMgBr}$. Following the general procedure, the reaction of $3 \mathrm{a}(110 \mathrm{mg}, 0.34 \mathrm{mmol})$ and $\mathrm{Me}_{2}$ CHMgBr $(1.2 \mathrm{~mL}$ of a $1.0 \mathrm{M}$ solution in ether, $1.2 \mathrm{mmol})$ in benzene gave $4 \mathrm{~b}(96 \mathrm{mg}, 77 \%$, de $48 \%)$. (2S,3S,1'S)-4b: $[\alpha]^{26}{ }_{\mathrm{D}}$ $=-50.3^{\circ}\left(c 1.5, \mathrm{CHCl}_{3}\right) ; \mathrm{IR}$ (neat) $v 3477,2974,2932,2874$ 


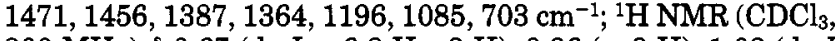
$300 \mathrm{MHz}) \delta 0.67(\mathrm{~d}, J=6.8 \mathrm{~Hz}, 3 \mathrm{H}), 0.96(\mathrm{~s}, 9 \mathrm{H}), 1.03(\mathrm{~d}, J$ $=6.6 \mathrm{~Hz}, 3 \mathrm{H}), 1.21(\mathrm{~s}, 9 \mathrm{H}), 1.89-2.01(\mathrm{~m}, 1 \mathrm{H}), 2.94(\mathrm{~d}, J=$ $5.8 \mathrm{~Hz}, 1 \mathrm{H}$ ), 3.07 (dd, $J=9.2,4.7 \mathrm{~Hz}, 1 \mathrm{H}), 3.26(\mathrm{dd}, J=9.2$, $7.1 \mathrm{~Hz}, 1 \mathrm{H}), 3.44-3.50(\mathrm{~m}, 2 \mathrm{H}), 3.55$ (dd, $J=8.9,4.9 \mathrm{~Hz}, 1$ $\mathrm{H}), 3.83-3.91(\mathrm{~m}, 1 \mathrm{H}), 4.02(\mathrm{~d}, J=7.6 \mathrm{~Hz}, 1 \mathrm{H}), 7.19-7.32$ $(\mathrm{m}, 5 \mathrm{H}) ;{ }^{13} \mathrm{C} \mathrm{NMR}\left(\mathrm{CDCl}_{3}, 75 \mathrm{MHz}\right) \delta 19.0,19.4,27.1,27.6$, $34.8,61.9,62.9,71.7,72.9,73.1,76.7,88.3,127.4,127.7,127.8$, $141.9 ; \mathrm{MS}(20 \mathrm{eV}) \mathrm{m} / z$ (rel intensity) $367(\mathrm{M}+1,100), 311$ (16), 261 (6), 235 (19), 211 (16), $179(29), 133(71), 107(20), 91$ (20), 57 (66); HRMS calcd for $\mathrm{C}_{22} \mathrm{H}_{38} \mathrm{O}_{4} 366.2770$, found 366.2777. Characteristic absorption for $\left(2 S, 3 S, 1^{\prime} R\right)-4 \mathbf{b}:{ }^{1} \mathrm{H}$ NMR $\left(\mathrm{CDCl}_{3}, 300 \mathrm{MHz}\right) \delta 4.07(\mathrm{~d}, J=8.1 \mathrm{~Hz}, 1 \mathrm{H})$.

Reaction of $3 \mathrm{~b}$ with $\mathrm{Me}_{2} \mathrm{CHMgBr}$. Following the general procedure, the reaction of $3 \mathbf{b}(102 \mathrm{mg}, 0.25 \mathrm{mmol})$ and $\mathrm{Me}_{2}-$ CHMgBr $(1.0 \mathrm{~mL}$ of a $1.0 \mathrm{M}$ solution in ether, $1.0 \mathrm{mmol})$ in benzene gave $4 \mathrm{c}(89 \mathrm{mg}, 79 \%$, de $71 \%)$. (2S,3S, $\left.1^{\prime} S\right)-4 \mathrm{c}:[\alpha]^{24} \mathrm{D}$ $=-52.1^{\circ}$ (c 2.0, $\mathrm{CHCl}_{3}$ ); IR (neat) $v 3496,2972,2877,1602$, $1587,1468,1366,1261,1153,1086,901,785,703 \mathrm{~cm}^{-1} ;{ }^{1} \mathrm{H}$ NMR $\left(\mathrm{CDCl}_{3}, 300 \mathrm{MHz}\right) \delta 0.66(\mathrm{~d}, J=6.8 \mathrm{~Hz}, 3 \mathrm{H}), 0.71(\mathrm{~d}, J$ $=6.8 \mathrm{~Hz}, 3 \mathrm{H}), 0.75(\mathrm{~d}, J=6.8 \mathrm{~Hz}, 3 \mathrm{H}), 0.84(\mathrm{~s}, 3 \mathrm{H}), 0.85(\mathrm{~s}$, $3 \mathrm{H}), 0.87(\mathrm{~d}, J=6.8 \mathrm{~Hz}, 6 \mathrm{H}), 1.03(\mathrm{~d}, J=6.6 \mathrm{~Hz}, 3 \mathrm{H}), 1.09$ (s, $6 \mathrm{H}$ ), 1.55 (septet, $J=6.8 \mathrm{~Hz}, 1 \mathrm{H}$ ), 1.80 (septet, $J=6.8$ $\mathrm{Hz}, 1 \mathrm{H}), 1.88-1.99(\mathrm{~m}, 1 \mathrm{H}), 2.92(\mathrm{~d}, J=5.6 \mathrm{~Hz}, 1 \mathrm{H}), 3.05$ (dd, $J=9.2,4.4 \mathrm{~Hz}, 1 \mathrm{H}$ ), 3.24 (dd, $J=9.2,7.2 \mathrm{~Hz}, 1 \mathrm{H}$ ), $3.40-$ $3.47(\mathrm{~m}, 2 \mathrm{H}), 3.49$ (dd, $J=9.0,5.3 \mathrm{~Hz}, 1 \mathrm{H}), 3.77(\mathrm{~s}, 3 \mathrm{H})$, $3.81-3.90(\mathrm{~m}, 1 \mathrm{H}), 3.97(\mathrm{~d}, J=7.8 \mathrm{~Hz}, 1 \mathrm{H}), 6.75-6.85(\mathrm{~m}, 3$ $\mathrm{H}), 7.19(\mathrm{t}, J=7.8 \mathrm{~Hz}, 1 \mathrm{H}) ;{ }^{13} \mathrm{C} \mathrm{NMR}\left(\mathrm{CDCl}_{3}, 75 \mathrm{MHz}\right) \delta$ $17.3,17.5,17.6,19.0,19.6,21.5,21.8,22.2,34.7,35.6,35.8$, $55.1,61.1,61.8,71.8,76.6,77.2,88.5,112.8,113.2,120.3,128.8$, 143.7, 159.3; MS $(20 \mathrm{eV}) \mathrm{m} / z$ (rel intensity) $452(\mathrm{M}, 11), 409$ (9), 368 (4), 325 (13), 283 (8), 241 (100), 179 (4), 163 (33), 137 (8); HRMS calcd for $\mathrm{C}_{27} \mathrm{H}_{48} \mathrm{O}_{5} 452.3502$, found 452.3502 . Characteristic absorption for $\left(2 S, 3 S, 1^{\prime} R\right)-4 \mathrm{c}:{ }^{1} \mathrm{H} \mathrm{NMR}\left(\mathrm{CDCl}_{3}\right.$, $300 \mathrm{MHz}$ ) $\delta 4.05(\mathrm{~d}, J=8.3 \mathrm{~Hz}, 1 \mathrm{H})$.

Reaction of $3 \mathrm{c}$ with $\mathbf{c}-\mathrm{C}_{5} \mathbf{H}_{9} \mathrm{MgCl}$. Following the general procedure, the reaction of $3 \mathrm{c}^{9}(119 \mathrm{mg}, 0.5 \mathrm{mmol})$ and $\mathrm{c}-\mathrm{C}_{5} \mathrm{H}_{9^{-}}$ $\mathrm{MgCl}(2.0 \mathrm{~mL}$ of a $1.0 \mathrm{M}$ solution in ether, $2.0 \mathrm{mmol})$ in benzene gave $4 \mathrm{~d}(126 \mathrm{mg}, 82 \%$, de $64 \%)$. (2S,3S,1'S)-4d: [ $\alpha]^{27} \mathrm{D}$ $=-61.4^{\circ}\left(c 1.2, \mathrm{CHCl}_{3}\right)$; IR (neat) $v 3472,2952,2871,1453$, $1309,1197,1120,1080,764,703 \mathrm{~cm}^{-1} ;{ }^{1} \mathrm{H} \mathrm{NMR}\left(\mathrm{CDCl}_{3}, 300\right.$ MHz) $\delta 0.94-1.07(\mathrm{~m}, 1 \mathrm{H}), 1.15-1.26(\mathrm{~m}, 1 \mathrm{H}), 1.37-1.69$ $(\mathrm{m}, 5 \mathrm{H}), 1.92-2.00(\mathrm{~m}, 1 \mathrm{H}), 2.15-2.29(\mathrm{~m}, 1 \mathrm{H}), 2.73(\mathrm{~d}, J=$ $5.4 \mathrm{~Hz}, 1 \mathrm{H}), 3.03(\mathrm{~s}, 3 \mathrm{H}), 3.05(\mathrm{dd}, J=10.1,4.3 \mathrm{~Hz}, 1 \mathrm{H})$, 3.13 (dd, $J=10.1,6.0 \mathrm{~Hz}, 1 \mathrm{H}), 3.39(\mathrm{~s}, 3 \mathrm{H}), 3.44-3.53(\mathrm{~m}, 2$ $\mathrm{H}), 3.57$ (dd, $J=9.8,4.9 \mathrm{~Hz}, 1 \mathrm{H}), 3.89-3.97(\mathrm{~m}, 1 \mathrm{H}), 4.04(\mathrm{~d}$, $J=9.0 \mathrm{~Hz}, 1 \mathrm{H}), 7.22-7.32(\mathrm{~m}, 5 \mathrm{H}) ;{ }^{13} \mathrm{C} \mathrm{NMR}\left(\mathrm{CDCl}_{3}, 50\right.$ $\mathrm{MHz}) \delta 25.0,25.3,29.3,30.6,47.3,58.8,59.1,70.8,72.3,73.5$, $76.4,88.4,127.5,127.6,128.1,142.7$; MS $(20 \mathrm{eV}) \mathrm{m} / z$ (rel intensity) $309(\mathrm{M}+1,8), 239$ (34), $159(47), 151(100), 133$ (12), 115 (16), 101 (14), 91 (34); HRMS calcd for $\mathrm{C}_{18} \mathrm{H}_{28} \mathrm{O}_{4}$ 308.1988, found 308.1988. Characteristic absorption for $\left(2 S, 3 S, 1^{\prime} R\right)-4 \mathrm{~d}:{ }^{1} \mathrm{H} \mathrm{NMR}\left(\mathrm{CDCl}_{3}, 300 \mathrm{MHz}\right) \delta 4.13(\mathrm{~d}, J=9.2$ $\mathrm{Hz}, 1 \mathrm{H})$.

Reaction of 3a with $\mathbf{c}-\mathrm{C}_{5} \mathbf{H}_{9} \mathbf{M g C l}$. Following the general procedure, the reaction of $3 \mathbf{a}(161 \mathrm{mg}, 0.5 \mathrm{mmol})$ and $\mathrm{c}-\mathrm{C}_{5} \mathrm{H}_{9-}$ $\mathrm{MgCl}(2.0 \mathrm{~mL}$ of a $1.0 \mathrm{M}$ solution in ether, $2.0 \mathrm{mmol})$ in benzene gave $4 \mathbf{e}(163 \mathrm{mg}, 83 \%$, de $96 \%)$. $\left(2 S, 3 S, 1^{\prime} S\right)-4 \mathrm{e}:[\alpha]^{23} \mathrm{D}$ $=-53.2^{\circ}$ (c 2.2, $\mathrm{CHCl}_{3}$ ); IR (neat) v 3495, 2973, 2871, 1474, $1454,1389,1364,1196,1085,881,763,703 \mathrm{~cm}^{-1} ;{ }^{1} \mathrm{H}$ NMR $\left(\mathrm{CDCl}_{3}, 300 \mathrm{MHz}\right) \delta 0.94(\mathrm{~s}, 9 \mathrm{H}), 0.94-1.08(\mathrm{~m}, 1 \mathrm{H}), 1.15-$ $1.26(\mathrm{~m}, 10 \mathrm{H}$, embodied a singlet $1.21,9 \mathrm{H}), 1.37-1.68(\mathrm{~m}, 5$ $\mathrm{H}), 1.88-1.99(\mathrm{~m}, 1 \mathrm{H}), 2.13-2.26(\mathrm{~m}, 1 \mathrm{H}), 2.94(\mathrm{br} \mathrm{s}, 1 \mathrm{H})$, $3.02(\mathrm{dd}, J=9.2,4.8 \mathrm{~Hz}, 1 \mathrm{H}), 3.21(\mathrm{dd}, J=9.2,7.3 \mathrm{~Hz}, 1 \mathrm{H})$ $3.43-3.49(\mathrm{~m}, 2 \mathrm{H}), 3.54(\mathrm{dd}, J=9.0,4.7 \mathrm{~Hz}, 1 \mathrm{H}), 3.82-3.90$ $(\mathrm{m}, 1 \mathrm{H}), 4.07(\mathrm{~d}, J=8.9 \mathrm{~Hz}, 1 \mathrm{H}), 7.19-7.29(\mathrm{~m}, 5 \mathrm{H}) ;{ }^{13} \mathrm{C}$ $\mathrm{NMR}\left(\mathrm{CDCl}_{3}, 75 \mathrm{MHz}\right) \delta 24.9,25.2,27.0,27.5,29.2,30.3,47.2$, $61.6,62.9,71.5,72.8,72.9,76.6,87.5,127.3,127.4,127.9,142.8$; MS $(20 \mathrm{eV}) \mathrm{m} / z$ (rel intensity) $393(\mathrm{M}+1,1), 323(2), 267(4)$, 235 (4), 211 (27), 159 (96), 129 (19), 117 (22), 107 (25), 91 (39), 57 (100); HRMS calcd for $\mathrm{C}_{24} \mathrm{H}_{40} \mathrm{O}_{4} 392.2927$, found 392.2934 . Characteristic absorption for $\left(2 S, 3 S, 1^{\prime} R\right)-4$ e: ${ }^{1} \mathrm{H} \mathrm{NMR}\left(\mathrm{CDCl}_{3}\right.$, $300 \mathrm{MHz}) \delta 4.17(\mathrm{~d}, J=9.0 \mathrm{~Hz}, 1 \mathrm{H})$.

(9) Normant, J. F.; Alexakis, A.; Ghribi, A.; Mangeney, P. Tetrahedron $1989,45,507$.
Reaction of $3 \mathrm{~d}$ with $\mathbf{c}-\mathrm{C}_{5} \mathbf{H}_{9} \mathbf{M g C l}$. Following the general procedure, the reaction of $3 \mathrm{~d}(100 \mathrm{mg}, 0.25 \mathrm{mmol})$ and $\mathrm{c}-\mathrm{C}_{5} \mathrm{H}_{9}-$ $\mathrm{MgCl}(1.0 \mathrm{~mL}$ of a $1.0 \mathrm{M}$ solution in ether, $1.0 \mathrm{mmol})$ in benzene gave $4 f(99 \mathrm{mg}, 84 \%$, de $95 \%)$. (2S,3S,1'S)-4f: $[\alpha]^{28}{ }_{\mathrm{D}}$ $=-54.9^{\circ}$ (c 1.7, $\mathrm{CHCl}_{3}$ ); IR (neat) v 3484, 2972, 2871, 1486, $1389,1363,1196,1084,766,737,698 \mathrm{~cm}^{-1} ;{ }^{1} \mathrm{H} \mathrm{NMR}\left(\mathrm{CDCl}_{3}\right.$, $300 \mathrm{MHz}) \delta 0.96(\mathrm{~s}, 9 \mathrm{H}), 1.02-1.16(\mathrm{~m}, 1 \mathrm{H}), 1.23(\mathrm{~s}, 9 \mathrm{H})$, $1.23-1.34(\mathrm{~m}, 1 \mathrm{H}), 1.38-1.68(\mathrm{~m}, 5 \mathrm{H}), 1.92-2.02(\mathrm{~m}, 1 \mathrm{H})$, $2.18-2.31(\mathrm{~m}, 1 \mathrm{H}), 2.96(\mathrm{~d}, J=5.3 \mathrm{~Hz}, 1 \mathrm{H}), 3.07$ (dd, $J=$ $9.2,4.7 \mathrm{~Hz}, 1 \mathrm{H}$ ), 3.26 (dd, $J=9.2,7.1 \mathrm{~Hz}, 1 \mathrm{H}$ ), 3.46-3.52 $(\mathrm{m}, 2 \mathrm{H}), 3.56(\mathrm{dd}, J=9.0,4.8 \mathrm{~Hz}, 1 \mathrm{H}), 3.84-3.93(\mathrm{~m}, 1 \mathrm{H})$, $4.13(\mathrm{~d}, J=8.9 \mathrm{~Hz}, 1 \mathrm{H}), 7.29-7.44(\mathrm{~m}, 5 \mathrm{H}), 7.51-7.58(\mathrm{~m}, 4$ $\mathrm{H}) ;{ }^{13} \mathrm{C} \mathrm{NMR}\left(\mathrm{CDCl}_{3}, 75 \mathrm{MHz}\right) \delta 25.0,25.3,27.1,27.6,29.4$, $30.4,47.2,61.8,62.9,71.7,72.9,73.0,76.8,87.2,126.7,127.0$, $127.1,128.0,128.7,140.3,141.0,142.0 ;$ MS $(20 \mathrm{eV}) \mathrm{m} / z$ (rel intensity) $468(\mathrm{M}, 0.04), 399(60), 343$ (14), 287 (100), 251 (8), 235 (25), 183 (25), 167 (13); HRMS calcd for $\mathrm{C}_{30} \mathrm{H}_{44} \mathrm{O}_{4} 468.3240$, found 468.3229 . Characteristic absorption for $\left(2 S, 3 S, 1^{\prime} R\right)-4 \mathbf{f}$ : ${ }^{1} \mathrm{H} \mathrm{NMR}\left(\mathrm{CDCl}_{3}, 300 \mathrm{MHz}\right) \delta 4.25(\mathrm{~d}, J=9.0 \mathrm{~Hz}, 1 \mathrm{H})$.

Reaction of $3 e$ with $\mathbf{c}-\mathrm{C}_{5} \mathrm{H}_{9} \mathrm{MgCl}$. Following the general procedure, the reaction of $3 e(114 \mathrm{mg}, 0.25 \mathrm{mmol})$ and c- $\mathrm{C}_{5} \mathrm{H}_{9-}$ $\mathrm{MgCl}(1.0 \mathrm{~mL}$ of a $1.0 \mathrm{M}$ solution in ether, $1.0 \mathrm{mmol})$ in benzene gave $\mathbf{4 g}(102 \mathrm{mg}, 78 \%$, de $96 \%)$. (2S,3S,1'S)-4g: $[\alpha]^{29}{ }_{\mathrm{D}}$ $=-49.3^{\circ}\left(c 4.3, \mathrm{CHCl}_{3}\right)$; IR (neat) v 3499, 2963, 2874, 1485, $1389,1366,1171,1081,766,737,698 \mathrm{~cm}^{-1} ;{ }^{1} \mathrm{H} \mathrm{NMR}\left(\mathrm{CDCl}_{3}\right.$, $300 \mathrm{MHz}) \delta 0.70(\mathrm{~d}, J=6.9 \mathrm{~Hz}, 3 \mathrm{H}), 0.74(\mathrm{~d}, J=6.9 \mathrm{~Hz}, 3$ $\mathrm{H}), 0.83(\mathrm{~s}, 6 \mathrm{H}), 0.90(\mathrm{~d}, J=6.8 \mathrm{~Hz}, 6 \mathrm{H}), 1.03-1.14(\mathrm{~m}, 7 \mathrm{H}$, embodied a singlet at $1.12,6 \mathrm{H}), 1.24-1.69(\mathrm{~m}, 7 \mathrm{H}), 1.83$ (septet, $J=6.8 \mathrm{~Hz}, 1 \mathrm{H}), 1.92-2.02(\mathrm{~m}, 1 \mathrm{H}), 2.19-2.32(\mathrm{~m}, 1$ H), 2.91 (d, $J=5.7 \mathrm{~Hz}, 1 \mathrm{H}), 3.02(\mathrm{dd}, J=9.2,4.4 \mathrm{~Hz}, 1 \mathrm{H}$ ), 3.24 (dd, $J=9.2,7.3 \mathrm{~Hz}, 1 \mathrm{H}), 3.43-3.55(\mathrm{~m}, 3 \mathrm{H}), 3.85-3.92$ $(\mathrm{m}, 1 \mathrm{H}), 4.14(\mathrm{~d}, J=8.9 \mathrm{~Hz}, 1 \mathrm{H}), 7.29-7.45(\mathrm{~m}, 5 \mathrm{H}), 7.51-$ $7.59(\mathrm{~m}, 4 \mathrm{H}) ;{ }^{13} \mathrm{C} \mathrm{NMR}\left(\mathrm{CDCl}_{3}, 75 \mathrm{MHz}\right) \delta 17.3,17.5,17.6$, $21.5,21.8,22.1,22.2,25.0,25.3,29.4,30.4,35.6,35.9,47.1$, $60.9,61.9,71.7,76.6,77.2,87.4,126.7,127.0,127.1,128.0$ $128.7,140.4,141.0,141.9 ; \mathrm{MS}(20 \mathrm{eV}) \mathrm{m} / z$ (rel intensity) 525 $(\mathrm{M}+1,0.1), 455(8), 371(4), 355(8), 287(100), 252$ (16), 235 (81), $207(5), 183$ (49), 167 (48), 85 (86); HRMS calcd for $\mathrm{C}_{34} \mathrm{H}_{52} \mathrm{O}_{4}$ 524.3866, found 524.3861. Characteristic absorption for $\left(2 S, 3 S, 1^{\prime} R\right)-4 \mathrm{~g}:{ }^{1} \mathrm{H} \mathrm{NMR}\left(\mathrm{CDCl}_{3}, 300 \mathrm{MHz}\right) \delta 4.25(\mathrm{~d}, J=$ $9.1 \mathrm{~Hz}, 1 \mathrm{H})$.

Reaction of $3 f$ with $\mathrm{c}_{-} \mathrm{C}_{5} \mathrm{H}_{9} \mathrm{MgCl}$. Following the general procedure, the reaction of $3 f(100 \mathrm{mg}, 0.25 \mathrm{mmol})$ and $\mathrm{c}-\mathrm{C}_{5} \mathrm{H}_{9-}$ $\mathrm{MgCl}(1.0 \mathrm{~mL}$ of a $1.0 \mathrm{M}$ solution in ether, $1.0 \mathrm{mmol})$ in benzene gave $4 \mathrm{~h}(91 \mathrm{mg}, 77 \%$, de $95 \%)$. (2S,3S,1'S)-4h: $[\alpha]^{23} \mathrm{D}$ $=-51.2^{\circ}$ (c 2.5, $\mathrm{CHCl}_{3}$ ); IR (neat) v 3482, 2972, 2871, 1591, $1485,1364,1196,1083,1011,880,820,755 \mathrm{~cm}^{-1}$; ${ }^{1} \mathrm{H}$ NMR $\left(\mathrm{CDCl}_{3}, 300 \mathrm{MHz}\right) \delta 0.94-1.06(\mathrm{~m}, 10 \mathrm{H}$, embodied a singlet $0.97,9 \mathrm{H}), 1.16-1.27(\mathrm{~m}, 10 \mathrm{H}$, embodied a singlet $1.20,9 \mathrm{H})$, $1.36-1.63(\mathrm{~m}, 5 \mathrm{H}), 1.84-1.94(\mathrm{~m}, 1 \mathrm{H}), 2.05-2.19(\mathrm{~m}, 1 \mathrm{H})$, $2.91(\mathrm{~d}, J=4.7 \mathrm{~Hz}, 1 \mathrm{H}), 3.05$ (dd, $J=9.3,4.9 \mathrm{~Hz}, 1 \mathrm{H}$ ), 3.23 (dd, $J=9.3,6.6 \mathrm{~Hz}, 1 \mathrm{H}), 3.41-3.47(\mathrm{~m}, 2 \mathrm{H}), 3.51$ (dd, $J=$ $9.1,4.9 \mathrm{~Hz}, 1 \mathrm{H}), 3.80-3.88(\mathrm{~m}, 1 \mathrm{H}), 4.07(\mathrm{~d}, J=8.7 \mathrm{~Hz}, 1$ $\mathrm{H}), 7.17(\mathrm{~d}, J=8.3 \mathrm{~Hz}, 2 \mathrm{H}), 7.41(\mathrm{~d}, J=8.3 \mathrm{~Hz}, 2 \mathrm{H}) ;{ }^{13} \mathrm{C}$ $\mathrm{NMR}\left(\mathrm{CDCl}_{3}, 75 \mathrm{MHz}\right) \delta 25.0,25.2,27.1,27.6,29.2,30.1,47.3$, $61.8,62.7,71.9,72.9,73.0,76.9,86.4,121.1,129.2,131.0,142.1$; FAB-MS $m / z$ (rel intensity) $473\left(\mathbf{M}\left({ }^{81} \mathrm{Br}\right)^{+}+1,6\right), 471$ (M$\left.\left({ }^{79} \mathrm{Br}\right)^{+}+1,6\right), 291(10), 289(10), 239(95), 237(100), 179(50)$, 171 (41), 169 (41), 154 (26), 123 (42), 57 (68); HRMS calcd for $\mathrm{C}_{24} \mathrm{H}_{39} \mathrm{O}_{4}{ }^{79} \mathrm{Br} 470.2032$, found 470.2028 . Characteristic absorption for $\left(2 S, 3 S, 1^{\prime} R\right)-4 \mathrm{~h}$ : ${ }^{1} \mathrm{H} \mathrm{NMR}\left(\mathrm{CDCl}_{3}, 300 \mathrm{MHz}\right) \delta 4.18$ (d, $J=8.7 \mathrm{~Hz}, 1 \mathrm{H})$.

Reaction of $3 \mathrm{~g}$ with $\mathbf{c}-\mathrm{C}_{6} \mathbf{H}_{11} \mathbf{M g B r}$. Following the general procedure, the reaction of $3 \mathbf{g}(130 \mathrm{mg}, 0.37 \mathrm{mmol})$ and $\mathrm{c}-\mathrm{C}_{6} \mathrm{H}_{11^{-}}$ $\mathrm{MgBr}(1.5 \mathrm{~mL}$ of a $1.0 \mathrm{M}$ solution in ether, $1.5 \mathrm{mmol}$ ) in benzene gave $4 \mathbf{i}(129 \mathrm{mg}, 80 \%$, de $>98 \%)$. (2S,3S,1'S)-4i: $[\alpha]^{28}{ }_{\mathrm{D}}=-41.8^{\circ}$ (c 5.2, $\mathrm{CHCl}_{3}$ ); IR (neat) $v 3494,2973,2930$, $2853,1601,1587,1487,1364,1258,1196,1085,879 \mathrm{~cm}^{-1} ;{ }^{1} \mathrm{H}$ $\mathrm{NMR}\left(\mathrm{CDCl}_{3}, 300 \mathrm{MHz}\right) \delta 0.73-0.87(\mathrm{~m}, 1 \mathrm{H}), 0.94-1.26(\mathrm{~m}$, $23 \mathrm{H}$, embodied two singlets $0.97,9 \mathrm{H}$ and $1.21,9 \mathrm{H}$ ), $1.52-$ $1.78(\mathrm{~m}, 4 \mathrm{H}), 2.07-2.17(\mathrm{~m}, 1 \mathrm{H}), 2.95(\mathrm{~d}, J=5.6 \mathrm{~Hz}, 1 \mathrm{H})$, 3.09 (dd, $J=9.1,4.5 \mathrm{~Hz}, 1 \mathrm{H}), 3.26(\mathrm{dd}, J=9.1,7.1 \mathrm{~Hz}, 1 \mathrm{H}$ ), $3.42-3.49(\mathrm{~m}, 2 \mathrm{H}), 3.53(\mathrm{dd}, J=9.1,4.9 \mathrm{~Hz}, 1 \mathrm{H}), 3.78(\mathrm{~s}, 3$ H), $3.81-3.89(\mathrm{~m}, 1 \mathrm{H}), 4.00(\mathrm{~d}, J=8.1 \mathrm{~Hz}, 1 \mathrm{H}), 6.75-6.83$ $(\mathrm{m}, 3 \mathrm{H}), 7.19(\mathrm{t}, J=7.8 \mathrm{~Hz}, 1 \mathrm{H}) ;{ }^{13} \mathrm{C} \mathrm{NMR}\left(\mathrm{CDCl}_{3}, 75 \mathrm{MHz}\right)$ $\delta 26.0,26.5,27.1,27.6,29.3,30.1,44.3,55.1,62.0,62.9,71.8$, 
$72.9,73.1,87.7,112.8,113.2,120.3,128.8,143.7,159.3$; MS $(20 \mathrm{eV}) \mathrm{m} / z$ (rel intensity) $436(\mathrm{M}, 3), 353(8), 297(11), 241$ (100), 219 (7), 203 (25), 137 (32), 126 (11), 121 (12), 105 (6), 87 (4), 57 (9); HRMS calcd for $\mathrm{C}_{26} \mathrm{H}_{44} \mathrm{O}_{5} 436.3189$, found 436.3190 .

Reaction of $3 \mathrm{a}$ with $\mathrm{c}_{-} \mathrm{C}_{6} \mathrm{H}_{11} \mathrm{MgBr}$. Following the general procedure, the reaction of $3 \mathrm{a}(120 \mathrm{mg}, 0.37 \mathrm{mmol})$ and $\mathrm{c}-\mathrm{C}_{6} \mathrm{H}_{11^{-}}$ $\mathrm{MgBr}(1.5 \mathrm{~mL}$ of a $1.0 \mathrm{M}$ solution in ether, $1.5 \mathrm{mmol})$ in benzene gave $4 \mathbf{j}(127 \mathrm{mg}, 84 \%$, de $>98 \%)$. (2S,3S,1'S)-4j: $[\alpha]^{29}{ }_{\mathrm{D}}=-38.0^{\circ}$ (c 3.3, $\mathrm{CHCl}_{3}$; IR (neat) v 3487, 2974, 2925, $2854,1452,1389,1363,1196,1085,880,758,703 \mathrm{~cm}^{-1} ;{ }^{1} \mathrm{H}$ NMR $\left(\mathrm{CDCl}_{3}, 300 \mathrm{MHz}\right) \delta 0.74-0.86(\mathrm{~m}, 1 \mathrm{H}), 0.92-1.26(\mathrm{~m}$, $23 \mathrm{H}$, embodied two singlets $0.95,9 \mathrm{H}$ and $1.21,9 \mathrm{H}), 1.51-$ $1.76(\mathrm{~m}, 4 \mathrm{H}), 2.07-2.17(\mathrm{~m}, 1 \mathrm{H}), 2.93(\mathrm{~d}, J=5.4 \mathrm{~Hz}, 1 \mathrm{H})$, 3.05 (dd, $J=9.2,4.7 \mathrm{~Hz}, 1 \mathrm{H}), 3.24(\mathrm{dd}, J=9.2,7.1 \mathrm{~Hz}, 1 \mathrm{H}$ ), $3.41-3.49(\mathrm{~m}, 2 \mathrm{H}), 3.53(\mathrm{dd}, J=9.0,4.9 \mathrm{~Hz}, 1 \mathrm{H}), 3.81-3.89$ $(\mathrm{m}, 1 \mathrm{H}), 4.03(\mathrm{~d}, J=7.9 \mathrm{~Hz}, 1 \mathrm{H}), 7.18-7.31(\mathrm{~m}, 5 \mathrm{H}) ;{ }^{13} \mathrm{C}$ $\mathrm{NMR}\left(\mathrm{CDCl}_{3}, 75 \mathrm{MHz}\right) \delta 26.0,26.5,27.1,27.6,29.3,30.0,44.3$, $61.9,62.9,71.7,72.9,73.0,76.5,87.7,127.4,127.8,141.9$; MS $(20 \mathrm{eV}) \mathrm{m} / z$ (rel intensity) $407(\mathrm{M}+1,1), 323(4), 301(3), 267$ (10), $211(81), 189(10), 173(100), 147(10), 129(22), 117(18)$, 107 (34), 91 (30), 57 (52); HRMS calcd for $\mathrm{C}_{25} \mathrm{H}_{42} \mathrm{O}_{4} 406.3083$, found 406.3080 .

Reaction of $3 \mathrm{~g}$ with $\mathrm{C}_{6} \mathrm{H}_{5} \mathrm{MgBr}$. Following the general procedure, the reaction of $3 \mathrm{~g}(130 \mathrm{mg}, 0.37 \mathrm{mmol})$ and $\mathrm{C}_{6} \mathrm{H}_{5^{-}}$ $\mathrm{MgBr}$ (3.0 mL of a $0.5 \mathrm{M}$ solution in ether, $3.0 \mathrm{mmol}$ ) in

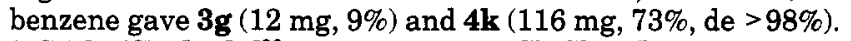
$\left(2 S, 3 S, 1^{\prime} S\right)-4 k:\left[\alpha{ }^{26}{ }_{\mathrm{D}}=+41.6^{\circ}\left(c 1.9, \mathrm{CHCl}_{3}\right)\right.$; IR (neat) $v 3482$, $2973,2934,1600,1587,1488,1364,1255,1195,1084,702$ $\mathrm{cm}^{-1} ;{ }^{1} \mathrm{H} \mathrm{NMR}\left(\mathrm{CDCl}_{3}, 300 \mathrm{MHz}\right) \delta 1.11(\mathrm{~s}, 9 \mathrm{H}), 1.12(\mathrm{~s}, 9 \mathrm{H})$, $2.84(\mathrm{~d}, J=4.9 \mathrm{~Hz}, 1 \mathrm{H}), 3.28(\mathrm{dd}, J=9.1,5.0 \mathrm{~Hz}, 1 \mathrm{H}), 3.37$ (dd, $J=9.1,6.9 \mathrm{~Hz}, 1 \mathrm{H}$ ), 3.49 (dd, $J=9.2,5.1 \mathrm{~Hz}, 1 \mathrm{H}$ ), 3.58$3.69(\mathrm{~m}, 2 \mathrm{H}), 3.75(\mathrm{~s}, 3 \mathrm{H}), 3.76-3.85(\mathrm{~m}, 1 \mathrm{H}), 5.65(\mathrm{~s}, 1 \mathrm{H})$, $6.76(\mathrm{~d}, J=8.2 \mathrm{~Hz}, 1 \mathrm{H}), 6.91-6.94(\mathrm{~m}, 2 \mathrm{H}), 7.17-7.36(\mathrm{~m}, 6$ $\mathrm{H}) ;{ }^{13} \mathrm{C} \mathrm{NMR}\left(\mathrm{CDCl}_{3}, 75 \mathrm{MHz}\right) \delta 27.3,27.5,55.1,62.3,63.1$, $71.7,72.9,73.2,76.3,82.6,112.4,112.8,119.5,127.6,128.3$, $129.1,142.1,144.6,159.5 ; \mathrm{MS}(20 \mathrm{eV}) \mathrm{m} / z$ (rel intensity) 430 (M, 3), $374(6), 372$ (12), 317 (4), 213 (98), 197 (100), 165 (18), 126 (9), 105 (9); HRMS calcd for $\mathrm{C}_{26} \mathrm{H}_{38} \mathrm{O}_{5} 430.2719$, found 430.2717 .

Reaction of $3 \mathrm{~g}$ with $\mathrm{Me}_{3} \mathrm{CCH}_{2} \mathrm{MgBr}$. Following the general procedure, the reaction of $3 \mathrm{~g}(97 \mathrm{mg}, 0.28 \mathrm{mmol})$ and $\mathrm{Me}_{3} \mathrm{CCH}_{2} \mathrm{MgBr}(1.2 \mathrm{~mL}$ of a $1.0 \mathrm{M}$ solution in ether, $1.2 \mathrm{mmol})$ in benzene gave 41 (95 mg, $81 \%$, de $94 \%)$. (2S,3S,1'S)-41: [ $\alpha]^{28}{ }_{\mathrm{D}}$ $=-63.5^{\circ}$ (c 1.0, $\mathrm{CHCl}_{3}$ ); IR (neat) $v 3500,2973,2872,1601$, $1589,1470,1364,1258,1196,1084,882,787,701 \mathrm{~cm}^{-1} ;{ }^{1} \mathrm{H}$ NMR $\left(\mathrm{CDCl}_{3}, 300 \mathrm{MHz}\right) \delta 0.87(\mathrm{~s}, 9 \mathrm{H}), 0.98(\mathrm{~s}, 9 \mathrm{H}), 1.20(\mathrm{~s}$, $9 \mathrm{H}$ ), 1.58 (dd, $J=14.3,5.6 \mathrm{~Hz}, 1 \mathrm{H}$ ), 1.86 (dd, $J=14.3,6.5$ $\mathrm{Hz}, 1 \mathrm{H}$ ), 2.93 (d, $J=5.1 \mathrm{~Hz}, 1 \mathrm{H}$ ), 3.09 (dd, $J=9.3,4.8 \mathrm{~Hz}$, $1 \mathrm{H}$ ), 3.22 (dd, $J=9.3,7.0 \mathrm{~Hz}, 1 \mathrm{H}$ ), $3.41-3.50(\mathrm{~m}, 2 \mathrm{H}$ ), 3.52 (dd, $J=9.0,4.7 \mathrm{~Hz}, 1 \mathrm{H}), 3.79(\mathrm{~s}, 3 \mathrm{H}), 3.81-3.89(\mathrm{~m}, 1 \mathrm{H})$, 4.49 (dd, $J=6.5,5.6 \mathrm{~Hz}, 1 \mathrm{H}), 6.75-6.79(\mathrm{~m}, 1 \mathrm{H}), 6.86-6.90$ $(\mathrm{m}, 2 \mathrm{H}), 7.20(\mathrm{t}, J=7.8 \mathrm{~Hz}, 1 \mathrm{H}) ;{ }^{13} \mathrm{C} \mathrm{NMR}\left(\mathrm{CDCl}_{3}, 75 \mathrm{MHz}\right)$ $\delta 27.2,27.6,30.2,51.7,55.2,62.2,62.9,71.9,72.9,73.1,75.9$, $80.4,112.8,112.9,119.8,129.2,146.1,159.6 ; \mathrm{MS}(20 \mathrm{eV}) \mathrm{m} / z$ (rel intensity) 424 (M, 17), $368(4), 311(3), 241(13), 235(100)$, 207 (14), 191 (34), 179 (39), 135 (29), 126 (14), 57 (30); HRMS calcd for $\mathrm{C}_{25} \mathrm{H}_{44} \mathrm{O}_{5} 424.3189$, found 424.3190 . Characteristic absorption for $\left(2 S, 3 S, 1^{\prime} R\right)-4 \mathrm{l}$ : ${ }^{1} \mathrm{H}$ NMR $\left(\mathrm{CDCl}_{3}, 300 \mathrm{MHz}\right) \delta$ 4.71 (dd, $J=7.8,4.6 \mathrm{~Hz}, 1 \mathrm{H}$ ).

Reaction of 7 with MeMgI. Following the general procedure, the reaction of $7(89 \mathrm{mg}, 0.5 \mathrm{mmol})$ and $\mathrm{MeMgI}(2.0$ $\mathrm{mL}$ of a $1.0 \mathrm{M}$ solution in ether, $2.0 \mathrm{mmol}$ ) in benzene gave 8 (80 $\mathrm{mg}, 82 \%$, 30\% de). The major isomer, $\left(2 S, 3 S, 1^{\prime} R\right)-8$, exhibited the spectroscopic data which are identical with those of the reported values. ${ }^{9}$

$\mathrm{TiCl}_{2}\left(\mathrm{O}^{\mathrm{P} P r}\right)_{2}$-Promoted Reaction of 3a with MeMgI. Following the general procedure, the reaction of $\mathbf{3 a}(161 \mathrm{mg}$,
$0.5 \mathrm{mmol}), \mathrm{MeMgI}(1.4 \mathrm{~mL}$ of a $2.0 \mathrm{M}$ solution in ether, 2.8 $\mathrm{mmol})$, and $\mathrm{TiCl}_{2}\left(\mathrm{O}^{\mathrm{PPr}}\right)_{2}(1.0 \mathrm{~mL}$ of a $1.0 \mathrm{M}$ solution in toluene, $1.0 \mathrm{mmol})$ in benzene at room temperature gave $4 \mathrm{a}(120 \mathrm{mg}$, $71 \%$, de $39 \%$ ).

Degradation of $4 \mathrm{a} . \mathrm{PCC}(862 \mathrm{mg}, 4.0 \mathrm{mmol})$ was suspended in $\mathrm{CH}_{2} \mathrm{Cl}_{2}(30 \mathrm{~mL})$, and the diastereomeric mixture of $4 a$ (338 $\mathrm{mg}, 1.0 \mathrm{mmol}, 26 \%$ de) was rapidly added. The mixture was stirred for $16 \mathrm{~h}$. The solvent was decanted, and the black solid was washed with $\mathrm{CH}_{2} \mathrm{Cl}_{2}(20 \mathrm{~mL} \times 2)$. The corresponding ketone $(265 \mathrm{mg}, 79 \%)$ was obtained by filtration of the organic extracts through Florisil and evaporation of the solvent in vacuo. Without purification, this ketone $(260 \mathrm{mg}, 0.77 \mathrm{mmol})$ was mixed with $m$-CPBA (172 $\mathrm{mg}, 1.0 \mathrm{mmol})$ in $\mathrm{CH}_{2} \mathrm{Cl}_{2}(20$ $\mathrm{mL})$, and the mixture was stirred for $8 \mathrm{~h} . \mathrm{Na}_{2} \mathrm{CO}_{3}(5 \%, 15$ $\mathrm{mL}$ ) was added, and the mixture was extracted with $\mathrm{CH}_{2} \mathrm{Cl}_{2}$ $(15 \mathrm{~mL} \times 2)$. The combined organic layers were washed with brine $(20 \mathrm{~mL})$ and dried $\left(\mathrm{MgSO}_{4}\right)$. The solvent was evaporated in vacuo, and the residue was chromatographed on silica gel (5\% ethyl acetate in hexane) to give a mixture of regioisomeric esters $(221 \mathrm{mg})$. Without purification, a mixture of the esters (216 $\mathrm{mg}, 0.61 \mathrm{mmol})$ and PPTS (10 mg) in MeOH (15 mL) was stirred for $4 \mathrm{~h}$, quenched with water $(8 \mathrm{~mL})$, and extracted with ether $(15 \mathrm{~mL} \times 2)$. The organic layer was evaporated in vacuo, and the residue was dissolved in $\mathrm{MeCN}(15 \mathrm{~mL})$ to which $\mathrm{HCl}(10 \%, 1.0 \mathrm{~mL})$ was added and the mixture was stirred for $12 \mathrm{~h}$. MeCN was removed in vacuo, and then additional water $(10 \mathrm{~mL})$ was introduced and extracted with ether $(15 \mathrm{~mL} \times 2)$. The organic layer was washed with brine $(15 \mathrm{~mL})$ and dried $\left(\mathrm{MgSO}_{4}\right)$. The solvent was removed in vacuo, and the residue was chromatographed on silica gel (30\% ether in pentane) to give the corresponding alcohol 5a $\left(\mathrm{R}^{1}=\right.$ $\mathrm{Ph}, \mathrm{R}^{2}=\mathrm{Me}, 35 \mathrm{mg}, 47 \%$ ) which exhibited physical properties identical to literature data: ${ }^{10}[\alpha]^{29}{ }_{\mathrm{D}}=-13.9^{\circ}\left(c 1.5, \mathrm{CH}_{2} \mathrm{Cl}_{2}\right)$ $\left[\right.$ lit. $\left.{ }^{10 \mathrm{~b}}(S)-5 \mathrm{a}[\alpha]^{22} \mathrm{D}=-52.5^{\circ}\left(\mathrm{c} 2.7, \mathrm{CH}_{2} \mathrm{Cl}_{2}\right)\right]$ which corresponds to $26 \%$ ee and was consistent with the estimation of the percent diastereomeric excess of the starting $4 \mathbf{a}$.

Degradation of $4 \mathrm{j}$. PCC (862 $\mathrm{mg}, 4.0 \mathrm{mmol}$ ) was suspended in $\mathrm{CH}_{2} \mathrm{Cl}_{2}$ (30 mL), and diastereomerically pure $4 \mathrm{j}$ (406 $\mathrm{mg}, 1.0 \mathrm{mmol}$ ) was rapidly added. The mixture was stirred for $20 \mathrm{~h}$. The solvent was decanted, and the black solid was washed with $\mathrm{CH}_{2} \mathrm{Cl}_{2}(20 \mathrm{~mL} \times 2)$. The corresponding ketone $(327 \mathrm{mg}, 81 \%$ ) was obtained by filtration of the organic extracts through Florisil and evaporation the solvent in vacuo. To a sodium $(230 \mathrm{mg}, 10 \mathrm{mmol})$ suspension in ether $(15 \mathrm{~mL})$ was added the ketone $(300 \mathrm{mg}, 0.74 \mathrm{mmol}$ ), and the mixture was stirred for $48 \mathrm{~h}$. The solvent was decanted and evaporated in vacuo. The residue was chromatographed on silica gel (10\% ethyl acetate in hexane) to give $5 b\left(R^{1}=P h, R^{2}=c-C_{6} H_{11}, 48\right.$ $\mathrm{mg}, 34 \%$ ) which exhibited physical properties identical to the literature data: $\left.{ }^{11}[\alpha]^{22} \mathrm{D}=-28.6^{\circ}(c) 1.2, \mathrm{C}_{6} \mathrm{H}_{6}\right)\left[\right.$ lit. $^{11}(S)-\mathbf{5 b}$ $\left.[\alpha]^{22_{\mathrm{D}}}=-28.3^{\circ}\left(\mathrm{c} 3.3, \mathrm{C}_{6} \mathrm{H}_{6}\right)\right]$.

Acknowledgment. Support from the National Science Council of the Republic of China is gratefully acknowledged.

Supplementary Material Available: ${ }^{1} \mathrm{H}$ NMR spectra for compounds 2a, 2b, 3a, 3b, 3d-g, and 4a-1 (21 pages). This material is contained in libraries on microfiche, immediately follows this article in the microfilm version of the journal, and can be ordered from the ACS; see any current masthead page for ordering information.

(10) Landor, S. R.; Chan, Y. M.; Sonola, O. O.; Tatchell, A. R. J. Chem. Soc., Perkin Trans. 1 1984, 493. (b) Nagai, U.; Shishido, T.; Chiba, R.; Mitsuhashi, H. Tetrahedron 1965, 21, 1701.

(11) Balfe, M. P.; Beaven, G. H.; Kenyon, J. J. Chem. Soc. 1950, 1857 . 